\title{
De novo genome assembly of Geosmithia morbida, the causal agent of thousand cankers disease
}

Taruna Aggarwal, Anthony Westbrook, Kirk Broders, Keith Woeste, Matthew D MacManes

Geosmithia morbida is a filamentous ascomycete that causes Thousand Cankers Disease in the eastern black walnut tree. This pathogen is commonly found in the western U.S.; however, recently the disease was also detected in several eastern states where the black walnut lumber industry is concentrated. G. morbida is one of two known phytopathogens within the genus Geosmithia, and it is vectored into the host tree via the walnut twig beetle. We present the first de novo draft genome of $G$. morbida. It is $26.5 \mathrm{Mbp}$ in length and contains less than $1 \%$ repetitive elements. The genome possesses an estimated 6,273 genes, 277 of which are predicted to encode proteins with unknown functions.

Approximately $31.5 \%$ of the proteins in G. morbida are homologous to proteins involved in pathogenicity, and $5.6 \%$ of the proteins contain signal peptides that indicate these proteins are secreted. Several studies have investigated the evolution of pathogenicity in pathogens of agricultural crops; forest fungal pathogens are often neglected because research efforts are focused on food crops. G. morbida is one of the few tree phytopathogens to be sequenced, assembled and annotated. The first draft genome of $G$. morbida serves as a valuable tool for comprehending the underlying molecular and evolutionary mechanisms behind pathogenesis within the Geosmithia genus.

Keywords: de novo genome assembly, pathogenesis, forest pathogen, black walnut, walnut twig beetle. 
1 De novo Genome Assembly of Geosmithia morbida, the Causal Agent of Thousand Cankers

2 Disease

3 Taruna Aggarwal, ${ }^{1 \star}$ Anthony Westbrook, ${ }^{2}$ Kirk Broders, ${ }^{3}$ Keith Woeste, ${ }^{4}$ Matthew D MacManes ${ }^{1}$

5 'Department of Molecular, Cellular, \& Biomedical Sciences, University of New Hampshire, 105

6 Main Street, Durham, NH 03824

$7 \quad$ 2Department of Computer Science, University of New Hampshire, 105 Main Street, Durham, NH

803824

9 3Department of Bioagricultural Sciences and Pest Management, Colorado State University, Fort

10 Collins, CO 80523

114 USDA Forest Service Hardwood Tree Improvement and Regeneration Center, Department of

12 Forestry and Natural Resources, Purdue University, West Lafayette, IN 47907

14 Corresponding author:

15 Taruna Aggarwal

1646 College Road, Durham, NH, 03824, United States

17 Email address: ta2007@wildcats.unh.edu 


\section{Abstract}

Geosmithia morbida is a filamentous ascomycete that causes Thousand Cankers Disease in the eastern black walnut tree. This pathogen is commonly found in the western U.S.; however, recently the disease was also detected in several eastern states where the black walnut lumber industry is concentrated. G. morbida is one of two known phytopathogens within the genus Geosmithia, and it is vectored into the host tree via the walnut twig beetle. We present the first de novo draft genome of G. morbida. It is $26.5 \mathrm{Mbp}$ in length and contains less than $1 \%$ repetitive elements. The genome possesses an estimated 6,273 genes, 277 of which are predicted to encode proteins with unknown functions. Approximately $31.5 \%$ of the proteins in $G$. morbida are homologous to proteins involved in pathogenicity, and $5.6 \%$ of the proteins contain putative signal peptides that indicate these proteins are secreted. Several studies have investigated the evolution of pathogenicity in pathogens of agricultural crops; forest fungal pathogens are often neglected because research efforts are focused on food crops. G. morbida is one of the few tree phytopathogens to be sequenced, assembled and annotated. The first draft genome of G. morbida serves as a valuable tool for comprehending the underlying molecular and evolutionary mechanisms behind pathogenesis within the Geosmithia genus.

\section{Introduction}

47 Studying molecular evolution of any phenotype is now made possible by the analysis of large amounts of sequence data generated by next-generation sequencing platforms. This is particularly beneficial for the study of emerging fungal pathogens, which are progressively recognized as a threat to global biodiversity and food security. Furthermore, in many cases their expansion is a result of anthropogenic activities and an increase in trade of fungal-infected goods [1]. Fungal pathogens are capable of evolving rapidly in order to overcome host 
53 resistance, fungicides, and to adapt to new hosts and environments. Whole genome sequence

54 data are useful in identifying the mechanisms of adaptive evolution within fungi [2-4]. For

55 instance, Stukenbrock et al. (2011) investigated the patterns of evolution in fungal pathogens

56 during the process of domestication in wheat using all aligned genes within the genomes of

57 wheat pathogens. They found that Zymoseptoria tritici, a domesticated wheat pathogen

58 (formerly known as Mycosphaerella graminicola), underwent adaptive evolution at a higher rate

59 than its wild relatives, Z. pseudotritici and Z. ardabiliae [5]. The study also revealed that many of

60 the pathogen's 802 secreted proteins were under positive selection. A study by Gardiner et al.

61 (2012), identified genes encoding aminotransferases, hydrolases, and kinases that were shared

62 between Fusarium pseudograminearum and other cereal pathogens. Using phylogenomic

63 analyses, the researchers demonstrated that these genes had bacterial origins. These studies

64 highlight the various evolutionary means that fungal species employ in order to adapt to specific

65 hosts, as well as the importance of genomics and bioinformatics in elucidating evolutionary

66 mechanisms within the fungal kingdom.

Many tree fungal pathogens associate with bark beetles in the family Scolytinae [6]. With

climate change, beetles and their fungal symbionts can invade new territory and become major invasive forest pests on a global scale [7, 8]. A well-known example of an invasive pest is the mountain pine beetle and its symbiont, Grosmannia clavigera that has affected approximately

713.4 million of acres of lodgepole, ponderosa, and five-needle pine trees in Colorado alone since

72 the outbreak began in $1996[9,10]$. Another beetle pest in the western U.S., Pityophthorous

73 juglandis (walnut twig beetle), associates with several fungal species, including the emergent

74 fungal pathogen Geosmithia morbida [11, 12].

Reports of tree mortality triggered by G. morbida infections first surfaced in 2009 [12], while the fungus was described as a new species in 2011 [11]. This fungus is vectored into the

77 host via $P$. juglandis and is the causal agent of thousand cankers disease (TCD) in Julgans

78 nigra (eastern black walnut) [13]. This walnut species is valued for its wood, which is used for 
79 furniture, cabinetry, and veneer. Although J. nigra trees are planted throughout western U.S. as

80 a decorative species, they are indigenous to eastern North America where the walnut industry is

81 worth hundreds of millions of dollars [13]. In addition to being a major threat to the eastern

82 populations of $\mathrm{J}$. nigra, TCD is of great concern because certain western walnut species

83 including J. regia (the Persian walnut), J. californica, and J. hindsii are also susceptible to the

84 fungus according to greenhouse inoculation studies [14].

85 The etiology of TCD is complex because it is a consequence of a fungal-beetle

86 symbiosis. The walnut twig beetle, which is only known to attack members of genera Juglans

87 and Pterocarya, is the most common vector of G. morbida [12]. Nevertheless, other beetles are

88 able to disperse the fungus from infested trees $[15,16]$. As vast numbers of beetles concentrate

89 in the bark of infested trees, fungal cankers form and coalesce around beetle galleries and

90 entrance holes. As the infection progresses, the phloem and cambium discolor and the leaves

91 wilt and yellow. These symptoms are followed by branch dieback and eventual tree death,

92 which can occur within three years of the initial infection [12]. Currently, 15 states in the U.S.

93 have reported one or more incidences of TCD, reflecting the expansion of WTB's geographic

94 range from its presumed native range in a few southwestern states [17]. Additionally, TCD has

95 also been found in Europe where walnut species are planted for timber [18].

96 To date, G. morbida is one of only two known pathogens within the genus Geosmithia,

97 which consists of mostly saprotrophic beetle-associated species (the other pathogen is $G$.

98 pallida) [19]. The ecological complexity this vector-host-pathogen system exhibits makes it an

99 intriguing lens for studying the evolution of pathogenicity. A well-assembled reference genome

100 will enable us to identify genes unique to G. morbida that may be utilized to develop sequence-

101 based tools for detecting and monitoring epidemics of TCD and for exploring the genomic

102 features of Geosmithia species, which may help explain the evolution of pathogenicity. Here, we

103 present a de novo genome assembly of Geosmithia morbida. The objectives of this study are to:

104 1) assemble the first, high-quality draft genome of this pathogen; 2) annotate the genome to 
105 better understand the genomic composition of Geosmithia species; and 3) briefly compare the

106 genome of G. morbida to two other fungal pathogens for which genomic data are available:

107 Fusarium solani, a root pathogen that infects soybean, and Grosmannia calvigera, a pathogenic 108 ascomycete that associates with the mountain pine beetle and kills lodgepole pines in North 109 America.

110 Methods

\section{DNA extraction and Library Preparation}

112 DNA was extracted using the CTAB method as outlined by the Joint Genome Institute to extract

113 DNA for Genome Sequencing from lyophilized mycelium of G. morbida (isolate 1262, host:

114 Juglans californica) from southwestern California [20]. The total DNA concentration was

115 measured using Nanodrop, and samples for sequencing were sent to Purdue University

116 Genomics Core Facility in West Lafayette, Indiana. DNA libraries were prepared using the

117 paired-end Illumina Truseq protocol and mate-pair Nextera DNA Sample Preparation kits with

118 average insert sizes of $487 \mathrm{bp}$ and $1921 \mathrm{bp}$, respectively. These libraries were sequenced on the

119 Illumina HiSeq 2500 using a single lane with a maximum read length of $101 \mathrm{bp}$.

\section{Preprocessing Sequence Data}

121 To assess the quality of our data, we ran FastQC (v0.11.2) (https://goo.gl/xHM1zf) [21] and

122 SGA Preqc (v0.10.13) (https://goo.gl/9y5bNy) on our raw sequence reads [22]. Both tools aim to

123 supply the user with information such as per base sequence quality score distribution (FastQC)

124 and frequency of variant branches in de Bruijn graphs (Preqc) that aid in selecting appropriate

125 assembly tools and parameters. The paired-end raw reads were corrected using a Bloom filter-

126 based error correction tool called BLESS (v0.16) (https://goo.gl/Kno6Xo) [23]. Next, the error

127 corrected reads were trimmed with Trimmomatic, version 0.32, using a Phred threshold of 2,

128 following recommendations from MacManes (2014) (https://goo.gl/FFoFjL)[24]. NextClip,

129 version 1.3.1, was leveraged to trim adapters in the mate-pair read set (https://goo.gl/aZ9ucT)

130 [25]. 
131 De novo genome assembly and evaluation

132 The de novo genome assembly was constructed with ALLPaths-LG (v49414)

133 (https://goo.gl/03gU9Z) [26]. The assembly was evaluated with BUSCO (v1.1b1)

134 (https://goo.gl/bMrXIM), a tool that assesses genome completeness based on the presence of

135 single-copy orthologs [27]. We also generated length-based statistics for our de novo genome

136 with QUAST (v2.3) (https://goo.gl/5KSa4M) [28]. The raw reads were mapped back to the

137 genome using BWA version 0.7.9a-r786 to further assess the quality of the assembly

138 (https://goo.gl/Scxgn4) [29].

\section{Structural and Functional Annotation of G. morbida genome}

140 We used the automated genome annotation software Maker version 2.31.8 [30]. Maker

141 identifies repetitive elements, aligns ESTs, and uses protein homology evidence to generate $a b$

142 initio gene predictions (https://goo.gl/JiLA3H). We used two of the three gene prediction tools

143 available within the pipeline, SNAP and Augustus. SNAP was trained using gff files generated

144 by CEGMA v2.5 (a program similar to BUSCO) [31]. Augustus was trained with Fusarium solani 145 protein models (v2.0.26) downloaded from Ensembl Fungi [32]. In order to functionally annotate

146 the genome, the protein sequences produced by the structural annotation were blasted against

147 the Swiss-Prot database, and target sequences were filtered for the best hits [33]. A small

148 subset of the resulting annotations was visualized and manually curated in WebApollo v2.0.1

149 [34]. The final annotations were also evaluated with BUSCO (v1.1b1) (https://goo.gl/thTGzH).

150 Assessing Repetitive Elements Profile

151 To assess the repetitive elements profile of $G$. morbida, we masked only the interspersed

152 repeats within the assembled scaffolds with RepeatMasker (v4.0.5) (https://goo.gl/TXrbr3) [35]

153 using the sensitive mode and default values as arguments. In order to compare the repetitive

154 element profile of G. morbida with F. solani (v2.0.29) and G. clavigera

155 (kw1407.GCA_000143105.2.30), the interspersed repeats of these two fungal pathogens were 
156 also masked with RepeatMasker. The genome and protein data of these fungi were downloaded

157 from Ensembl Fungi [32].

158 Identifying putative proteins contributing to pathogenicity

159 To identify putative genes contributing to pathogenicity in G. morbida, a BLASTp search was

160 conducted for single best hits at an e-value threshold of 1e-6 or less against the PHI-base

161 database (v3.8) (https://goo.gl/CEEVY0) that contains experimentally confirmed genes from

162 fungal, oomycete and bacterial pathogens [36]. The search was performed using the same

163 parameters for F. solani and G. clavigera. To identify the proteins that contain signal peptides,

164 we used SignalP (v4.1) (https://goo.gl/JOe5Dh), and compared results from G. morbida with

165 those from F. solani and G. clavigera [37]. Lastly, to find putative protein domains involved in

166 pathogenicity in G. morbida, we performed a HMMER (version 3.1b2) [38] search against the

167 Pfam database (v28.0) [39] using the protein sequences as query. We conducted the same

168 search for sequences of 17 known effector proteins, then extracted and analyzed domains

169 common between the effector sequences and G. morbida (https://goo.gl/Y9IPZs).

170 Data availability

171 The sequences reported in this manuscript are being submitted to the European Nucleotide

172 Archive under Project Number PRJEB13066.

173 Results and Discussion

174 Data Processing

175 A total of 28,027,726 paired-end (PE) and 41,348,578 mate-pair (MP) reads were generated

176 with approximately $109 x$ and $160 x$ coverage, respectively (Table 1 ). Of the MP reads, $67.7 \%$

177 contained adapters that were trimmed using NextClip (v1.3.1). We corrected errors within the

178 PE reads using BLESS (v0.16) at a kmer length of 21. After correction, low-quality reads (phred

179 score < 2) were trimmed with Trimmomatic (v0.32) resulting in $99.75 \%$ reads passing. In total,

$18016,336,158 \mathrm{MP}$ and 27,957,268 PE reads were used to construct the de novo genome

181 assembly. 
182 Table 1. Statistics for Geosmithia morbida sequence data. The values in bold are final number

183 of reads used for assembly after quality check.

\begin{tabular}{|c|c|c|}
\hline & Paired-end & Mate-pair \\
\hline Number of reads & $27,957,268$ & $41,348,578$ \\
\hline Average insert size (bp) & 487 & 1921 \\
\hline Average coverage & $109 x$ & $160 x$ \\
\hline
\end{tabular}

184

185

186

187

188

189

Assembly Features

190 The G. morbida de novo assembly was constructed with AllPaths-LG (v49414). The assembled

191 genome consisted of 73 contigs totaling $26,549,069 \mathrm{bp}$, which is comparable to certain other

192 Ascomycetes such as Acremonium chrysogenum and Ustilaginoidea virens with genome sizes

193 of 28.6 and 30.2 Mbp, respectively. The largest contig length was 2,597,956 bp, and the NG50

194 was $1,305,468 \mathrm{bp}$. The completeness of the genome assembly was assessed using BUSCO, a

195 tool that scans the genome for the presence of single-copy orthologous groups present in more

196 than $90 \%$ of fungal species. Of 1,438 single-copy orthologs specific to fungi, $98 \%$ were

197 complete in our assembly, and 4.3\% were duplicated BUSCOs. Only one ortholog was missing

198 from the genome (Table 2). We used BWA to map the unprocessed, raw MP and PE reads

199 back to the genome to further evaluate the assembly, and $87 \%$ of the MP and $90 \%$ of the PE

200 reads mapped to our reference genome.

201

202 
209 Table 2. Geosmithia morbida reference genome assembly statistics generated using QUAST 210 (v2.3).

\begin{tabular}{ll}
\hline & Scaffolds \\
\hline Number of sequences & 73 \\
Largest scaffold length & $2,597,956$ \\
N50 & $1,305,468$ \\
L50 & 7 \\
Total assembly length & $26,549,069$ \\
GC\% & 54.31 \\
BUSCOs completeness & $98 \%$ \\
\hline
\end{tabular}

\section{Gene annotation}

213 The automated genome annotation software Maker v2.31.8 was used to identify structural

214 elements in the G. morbida assembly generated by AllPaths-LG. Of the total 6,273 proteins that 215 were predicted, 5,996 had protein-homology evidence in the Swiss-Prot database and only 277 $216(4.41 \%)$ of the total genes encoded for proteins of unknown function. Even though the total of 2176,273 proteins is lower than the average number of 11,129 genes in Ascomycota, this number is 218 within the range of the 4,657 and 27,529 coding genes within the phylum [40]. The 219 completeness of the functional annotations was evaluated using BUSCO, and $95 \%$ of the single 220 copy orthologs were present in this protein set and only $7 \%$ were duplicated BUSCOs. 


\section{Repetitive Elements}

222 Repetitive elements represented $0.81 \%$ of the total bases in G. morbida. The genome contained

223152 retroelements (class I) that were mostly composed of long terminal repeats $(n=146)$ and 60

224 DNA transposons (class II). In comparison, the genomes of $G$. clavigera and $F$. solani contained

$2251.14 \%$ and $1.47 \%$, respectively. G. clavigera possesses 541 retroelements $(0.79 \%)$ and 66 DNA

226 transposons $(0.04 \%)$, whereas the genome of $F$. solani is comprised of $499(0.54 \%)$ and 515

$227(0.81 \%)$ retroelements and transposons, respectively. The larger number of repeat elements in

228 F. solani may explain its relatively large genome size -51.3 Mbp versus G. clavigera's 29.8

229 Mbp and G. morbida's 26.5 Mbp (Table 3).

230 Table 3. Repetitive elements profile for Geosmithia morbida, Grosmannia clavigera, and

231 Fusarium solani. RepeatMasker (v4.0.5) was used to generate these values. Genomic data for

232 F. solani and G. clavigera were downloaded from Ensembl Fungi.

\begin{tabular}{lccc}
\hline & G. morbida & G. clavigera & F. solani \\
\hline Genome size & $26.5 \mathrm{Mbp}$ & $29.8 \mathrm{Mbp}$ & $51.3 \mathrm{Mbp}$ \\
\% Repetitive element & $0.81 \%$ & $1.14 \%$ & $1.47 \%$ \\
\% Retroelements & $0.10 \%$ & $0.79 \%$ & $0.54 \%$ \\
\% DNA transposons & $0.02 \%$ & $0.04 \%$ & $0.81 \%$ \\
\hline
\end{tabular}

234 Identifying and classifying putative pathogenicity genes

235 We blasted the entire predicted protein set against the PHI-base database (v3.8) to identify a list

236 of putative genes that may contribute to pathogenicity within G. morbida, F. solani, and G.

237 clavigera. We determined that 1,974 genes in G. morbida ( $31.47 \%$ of the total 6,273 genes)

238 were homologous to protein sequences in the database (Table S1). For F. solani and G.

239 clavigera, there were 4,855 and 2,387 genes with homologous PHI-base proteins (Table S2and

240 Table S3).

241 Identifying putative secreted proteins 
242 A search for the presence of putative secreted peptides within the protein sequences of $G$.

243 morbida, F. solani and G. clavigera showed that approximately $5.6 \%$ (349) of the G. morbida

244 sequences contained signal peptides (Table S4). Of the 349 sequences containing putative

245 signal peptides, only 27 encoded proteins of unknown function. Roughly $8.8 \%$ and $6.9 \%$ of the

246 proteins of $F$. solani and G. clavigera possess signal peptides (Table S5 and Table S6).

247 Secreted proteins are essential for host-fungal interactions and are indicative of adaptation

248 within fungal pathogens that require an array of mechanisms to overcome plant host defenses.

249 Even though the precise means by which fungal proteins are trafficked into the host are unclear,

250 secreted proteins are known to be essential for the translocation of fungal proteins into the host

251 cells [41]. For instance, race 1 strains of Verticillium dahliae, a common cause of vascular wilt

252 disease in plants, secretes a protein called Ave1 that induces host immunity response

253 suggesting this protein is crucial for virulence [42]. Another example of a secreted protein is

254 Ecp6 in fungal pathogen Cladosporium fulvum that prevents chitin-activated detection by the

255 host plant [43].

\section{Identifying protein domains}

257 We conducted a HMMER search against the pfam database (v28.0) using amino acid

258 sequences for G. morbida and 17 effector proteins from various fungal species. For G. morbida,

259 there were 6,023 unique protein domains out of a total of 43,823 Pfam hits. A total of 17

260 domains, which comprised 1,000 hits, were shared between G. morbida and known effector

261 proteins. The three most common protein domains in G. morbida with a putative effector

262 function belonged to short-chain dehydrogenases $(n=111)$, polyketide synthases $(n=94)$ and

263 NADH dehydrogenases ( $n=86)$. The HMMER G. morbida and effector proteins output files can

264 be found in Table S7and Table S8.

\section{Conclusion}

266 This work introduces the first genome assembly and analysis of Geosmithia morbida, a fungal

267 pathogen of the black walnut tree that is vectored into the host via the walnut twig beetle. The 
268 de novo assembly is composed of 73 scaffolds totaling in $26.5 \mathrm{Mbp}$. There are 6,273 predicted 269 proteins, and $4.41 \%$ of these are unknown. In comparison, $68.27 \%$ of $F$. solani and $26.70 \%$ of

270 G. clavigera predicted proteins are unknown. We assessed the quality of our genome assembly

271 and the predicted protein set using BUSCO, and found that $98 \%$ and $95 \%$ of the single copy

272 orthologs specific to the fungal lineage were present in both, respectively. These data are

273 indicative of our assembly's high quality and completeness. Our BLASTp search against the

274 PHI-base database revealed that G. morbida possesses 1,974 genes that are homologous to

275 proteins involved in pathogenicity. Furthermore, G. morbida shares several domains with known

276 effector proteins that are key for fungal pathogens during the infection process.

277 Geosmithia morbida is one of only two known fungal pathogens within the Geosmithia

278 genus [19]. The genome assembly introduced in this study can be leveraged to explore the

279 molecular mechanisms behind pathogenesis within this genus. The putative list of pathogenicity

280 genes provided in this study can be used for future comparative genomic analyses, knock-out,

281 and inoculation experiments. Moreover, genes unique to G. morbida may be utilized to develop

282 DNA sequence-based tools for detecting and monitoring ongoing and future TCD epidemics.

\section{References}

284 1. Fisher MC, Henk DA, Briggs CJ, Brownstein JS, Madoff LC, McCraw SL, et al. Emerging 285 fungal threats to animal, plant and ecosystem health. Nature. 2012;484:186-194.

286 2. Stukenbrock EH, Bataillon T, Dutheil JY, Hansen TT, Li R, Zala M, et al. The making of a new pathogen: Insights from comparative population genomics of the domesticated wheat pathogen Mycosphaerella graminicola and its wild sister species. Genome Res. $2011 ; 21: 2157-2166$.

3. Gardiner DM, McDonald MC, Covarelli L, Solomon PS, Rusu AG, Marshall M, et al. Comparative Pathogenomics Reveals Horizontally Acquired Novel Virulence Genes in Fungi Infecting Cereal Hosts. PLoS Pathog. 2012;8:e1002952-22. 
293 4. Condon BJ, Leng Y, Wu D, Bushley KE, Ohm RA, Otillar R, et al. Comparative Genome Structure, Secondary Metabolite, and Effector Coding Capacity across Cochliobolus Pathogens. PLoS Genet. 2013;9:e1003233.

5. Stukenbrock EH, Quaedvlieg W, Javan-Nikhah M, Zala M, Crous PW, et al. Zymoseptoria ardabiliae and Z. pseudotritici, two progenitor species of the septoria tritici leaf blotch fungus Z. tritici. Mycologia. 2012;104(6):1397-1407.

6. Six DL, Wingfield MJ. The role of phytopathogenicity in bark beetle-fungus symbioses: A challenge to the classic paradigm. Annu Rev Entomol. 2011;56:255-272.

7. Kurz WA, Dymond CC, Stinson G, Rampley GJ, Neilson ET, Carroll AL, et al. Mountain pine beetle and forest carbon feedback to climate change. Nature. 2008;452:987-990.

8. Sambaraju KR, Carroll AL, Zhu J, Stahl K, Moore RD, Aukema BH. Climate change could alter the distribution of mountain pine beetle outbreaks in western Canada. Ecography. 2012;35:211-223.

9. Massoumi Alamouti S, Haridas S, Feau N, Robertson G, Bohlmann J, Breuil C. Comparative Genomics of the Pine Pathogens and Beetle Symbionts in the Genus Grosmannia. Mol Biol Evol. 2014;31:1454-1474.

10. Mountain Pine Beetle: http://csfs.colostate.edu/forest-management/common-forestinsects- diseases/mountain-pine-beetle/. Accessed 15 April 2015.

11. Tisserat N, Cranshaw W, Leatherman D, Utley C, Alexander K. Black Walnut Mortality in Colorado Caused by the Walnut Twig Beetle and Thousand Cankers Disease. PHP. 2009:1-10. doi:10.1094/PHP-2009-0811-01-RS 12. Kolarik M, Freeland E, Utley C, Tisserat N. Geosmithia morbida sp. nov., a new phytopathogenic species living in symbiosis with the walnut twig beetle (Pityophthorus juglandis) on Juglans in USA. Mycologia. 2011;103:325-332. 
13. Zerillo MM, Caballero JI, Woeste K, Graves AD, Hartel C, Pscheidt JW, et al. Population Structure of Geosmithia morbida, the Causal Agent of Thousand Cankers Disease of Walnut Trees in the United States. PLoS ONE. 2014;9(11):e112847.

14. Utley C, Nguyen T, Roubtsova T, Coggeshall M, Ford TM, Grauke LJ et al. Susceptibility of Walnut and Hickory Species to Geosmithia morbida. Plant Disease. 2013;97(5):601607.

15. Kolařík M, Kostovčík M, Pažoutová S. Host range and diversity of the genus Geosmithia (Ascomycota: Hypocreales) living in association with bark beetles in the Mediterranean area. Mycological Res. 2007;111:1298-1310.

16. Kolařík M, Jankowiak R. Vector Affinity and Diversity of Geosmithia Fungi Living on Subcortical Insects Inhabiting Pinaceae Species in Central and Northeastern Europe. Microb Ecol. 2013;66:682-700.

17. Rugman-Jones PF, Seybold SJ, Graves AD, Stouthamer R. Phylogeography of the Walnut Twig Beetle, Pityophthorus juglandis, the Vector of Thousand Cankers Disease in North American Walnut Trees. PLoS ONE. 2015;10:e0118264. morbida and Walnut Twig Beetle Pityophthorus juglandis on Juglans nigra in Europe. Plant Disease. 2014;98(5):696.

19. Lynch SC, Wang DH, Mayorquin JS, Rugman-Jones PF, Stouthamer R, Eskalen E. First Report of Geosmithia pallida Causing Foamy Bark Canker, a New Disease on Coast Live Oak (Quercus agrifolia), in Association with Pseudopityophthorus pubipennis in California. 2014; 98:1276.

20. Kohler A, Francis M. Genomic DNA Extraction. http://1000.fungalgenomes.org/home/wpcontent/uploads/2013/02/genomicDNAProtocol-AK0511.pdf. Accessed 12 Dec 2015.

21. Andrews S. FastQC. http://www.bioinformatics.babraham.ac.uk/projects/fastqc/. Accessed 12 Dec 2015. 
343 22. Simpson JT. Exploring genome characteristics and sequence quality without a 344 reference. arXiv. 2013; http://arxiv.org/abs/1307.8026.

345 23. Heo Y, Wu X-L, Chen D, Ma J, Hwu W-M. BLESS: bloom filter-based error correction 346 solution for high-throughput sequencing reads. Bioinformatics. 2014;30:1354-1362.

347 24. Bolger AM, Lohse M, Usadel B. Trimmomatic: a flexible trimmer for Illumina sequence 348 data. Bioinformatics. 2014;30:2114-2120.

349 25. Leggett RM, Clavijo BJ, Clissold L, Clark MD, Caccamo M. NextClip: an analysis and $350 \quad$ read preparation tool for Nextera long mate pair libraries. Bioinformatics.

$351 \quad 2014 ; 30(4): 566-568$.

352 26. Gnerre S, MacCallum I, Przybylski D, Ribeiro FJ, Burton JN, Walker BJ, et al. High353 quality draft assemblies of mammalian genomes from massively parallel sequence data. $354 \quad$ PNAS. 2011;108:1513-1518.

355 27. Simão FA, Waterhouse RM, loannidis P, Kriventseva EV, Zdobnov EM. BUSCO: assessing genome assembly and annotation completeness with single-copy orthologs. Bioinformatics. 2015:1-3 (2015).

28. Gurevich A, Saveliev V, Vyahhi N, Tesler G. QUAST: quality assessment tool for genome assemblies. Bioinformatics. 2013;29:1072-1075.

29. Li H, Durbin R. Fast and accurate short read alignment with Burrows-Wheeler transform. Bioinformatics. 2009;25:1754-1760. annotation pipeline designed for emerging model organism genomes. Genome Res. 2008;18:188-196.

31. Parra G, Bradnam K, Korf I. CEGMA: a pipeline to accurately annotate core genes in eukaryotic genomes. Bioinformatics. 2007;23:1061-1067.

32. EnsemblFungi: (http://fungi.ensembl.org/index.html). Accessed 14 Nov 2015.

33. Swiss-Prot: (http://www.uniprot.org/). Downloaded 6 May 2015. 
34. Lee E, Helt GA, Reese JT, Munoz-Torres MC, Childers CP, Buels RM, et al. Web Apollo: a web-based genomic annotation editing platform. Genome Biol. 2013;4:R93.

372 36. PHI-base: The Pathogen - Host Interaction Database: (http://www.phi-base. org/).

$373 \quad$ Accessed 22 Nov 2015.

374 37. Peterson TN, Brunak S, von Heijne G, Nielsen H. SignalP 4.0: discriminating signal 375 peptides from transmembrane regions. Nature Methods. 2011;8:785-786.

376 38. Finn RD, Clements J, Eddy SR. HMMER web server: interactive sequence similarity 377 searching. Nucl Acids Res. 2011:1-9.

378 39. Finn RD, Bateman A, Clements J, Coggill P, Eberhardt RY, Eddy SR, et al. Pfam: the 379 protein families database. Nucl Acids Res. 2014;42:D222-D230.

380 40. Mohanta TK, Bae H. The diversity of fungal genome. Biol. Proced. Online. 2015;17(8):1381 9.

41. Petre B, Kamoun S. How Do Filamentous Pathogens Deliver Effector Proteins into Plant Cells? PLoS Biol. 2014;12(2):1-7.

42. de Jonge R, van Esse HP, Maruthachalam K, Bolton MD, Santhanam $P$, et al. Tomato immune receptor Ve1 recognizes effector of multiple fungal pathogens uncovered by genome and RNA sequencing. PNAS. 2012;109(13):5110-5115.

43. de Jonge R, van Esse HP, Kombrink A, Shinya T, Desaki Y, et al. Conserved Fungal LysM Effector Ecp6 Prevents Chitin-Triggered Immunity in Plants. Science. 